\title{
Roberge-Weiss periodicity and confinement-deconfinement transition
}

\author{
Kouji Kashiwa* \\ $\dagger$ \\ Yukawa Institute for Theoretical Physics, Kyoto University, Kyoto 606-8502, Japan \\ E-mail: kouji.kashiwa@yukawa.kyoto-u.ac.jp
}

\section{Akira Ohnishi}

Yukawa Institute for Theoretical Physics, Kyoto University, Kyoto 606-8502, Japan

E-mail: ohnishi@yukawa.kyoto-u.ac.jp

\begin{abstract}
We propose a new determination of the confinement-deconfinement transition by using the imaginary chemical potential. The imaginary chemical potential can be interpreted as the AharonovBohm phase and then an analogy of the topological-order suggests that the Roberge-Weiss endpoint would define the deconfinement temperature. Based on the topological property, we can construct a new quantity which describes the confinement-deconfinement transition. This quantity is defined as the integral of the quark number susceptibility along the closed loop of $\theta$ where $\theta$ is the dimensionless imaginary chemical potential. Expected behavior of it at finite temperature is discussed and its asymptotic behaviors are shown.
\end{abstract}

34th annual International Symposium on Lattice Field Theory

24-30 July 2016

University of Southampton, UK

\footnotetext{
* Speaker.

${ }^{\dagger}$ This talk is based on paper [7].
} 


\section{Introduction}

Understanding the confinement-deconfinement transition in quantum chromodynamics (QCD) is one of the interesting subjects in nuclear physics, elementary particle physics and astrophysics. In the heavy quark mass limit, it is well known that the spontaneous center $\left(\mathbb{Z}_{N_{\mathrm{c}}}\right)$ symmetry breaking is directly related to the confinement-deconfinement transition, where $N_{\mathrm{c}}$ is the number of color. Then the Polyakov-loop becomes an exact order-parameter of the confinement-deconfinement transition. On the other hand, we cannot find any exact order-parameters in the case with dynamical quarks at present where the direct relation between $\mathbb{Z}_{N_{\mathrm{c}}}$ symmetry and the confinement-deconfinement transition is vanished.

Topological order - The notion of the topological order may be important to understand the nature of the confinement-deconfinement transition. Recently, the confined and deconfined states at zero temperature $(T=0)$ are classified by using the topological order [1] in Ref. [2]. In the classification, the degeneracy of the ground state plays a crucial role. Motivated by the progress, it has been suggested that the confinement-deconfinement transition at finite temperature $(T)$ can be described by using the analogy of the topological order. In the determination, the free-energy degeneracy plays a crucial role [3].

Imaginary chemical potential - In QCD at finite $T$, the imaginary chemical potential $\left(\mu_{\mathrm{I}}\right)$ is an external parameter related with the imaginary quark number density and shows the special periodicity. It is called the Roberge-Weiss (RW) periodicity [4] and its period is $2 \pi T / N_{\mathrm{c}}$. The RW periodicity leads the free-energy degeneracy and thus it is natural to think that some hints to understand the confinement-deconfinement transition are hidden in the imaginary chemical potential region.

Quark number holonomy - We investigate the confinement-deconfinement transition by using the imaginary chemical potential based on the analogy of the topological order. We discuss the contour integral along the closed loop of the dimensionless quark imaginary chemical potential, $\theta=\mu_{\mathrm{I}} / T$. Particularly, we focus on the behavior of the quark number density at finite $\mu_{\mathrm{I}}$ which is the $\theta$-odd quantity and propose a new (quantum) order-parameter which can describe the confinement-deconfinement transition even if dynamical quarks are acting in the system. We call it the quark number holonomy. The quark number holonomy seems to be a similar quantity with the Uhlmann phase [5, 6]. The Uhlmann phase can describe the topological order at finite $T$ at least in the one-dimensional fermion systems such as the topological insulator and the superconductor [6]. The Uhlmann phase is defined by using the amplitude for the density matrix where amplitudes form the Hilbert space. There is the $U(n)$ gauge freedom of the amplitude where $n$ is the dimension of the space and it is a generalization of the $U(1)$ gauge freedom of pure quantum states. Thus the Uhlmann phase is the extension of the Berry phase. At finite $T$, the Uhlmann phase includes information of the density matrix of the statistical mechanics and is calculated by the contour integral along the crystalline momentum. Unfortunately, the calculation of the Uhlmann phase in QCD seems to be very difficult or impossible at present. However, the quark number holonomy is calculable quantity in QCD and it also includes the information of the density matrix via the quark number density. It should be noted that the quark number holonomy can be easily calculated in the effective models of QCD and lattice QCD simulation as discussed later. It is the most important reason why we propose the new quantity for the confinement-deconfinement transition. 


\subsection{QCD periodicities and transitions at finite $\mu_{\mathrm{I}}$}

It is known that the $\mathrm{QCD}$ partition function $\left(Z_{\mathrm{QCD}}\right)$ has the RW periodicity [4];

$$
Z_{\mathrm{QCD}}(\theta)=Z_{\mathrm{QCD}}\left(\theta+\frac{2 \pi k}{N_{\mathrm{c}}}\right),
$$

where $k$ is any integer. The RW periodicity is an exact property of the QCD partition function. In the pure gauge limit $m \rightarrow \infty$, there is the $\mathbb{Z}_{N_{\mathrm{c}}}$ symmetry, but it is explicitly broken by dynamical quark effects. In addition to the RW periodicity, QCD has special transition at $\theta=(2 k-1) \pi / N_{\mathrm{c}}$ which is so called the RW transition. The RW periodicity is occurred via different ways in the confined and deconfined phases. Therefore, we can expect the endpoint of the RW transition line. The endpoint of the RW transition is called the RW endpoint and its temperature is denoted by $T_{\mathrm{RW}}$.

\subsection{Deconfinement transition from RW periodicity}

In Ref. [7], the authors proposed the new classification of the confined and deconfined phases at finite $T$ based on the RW periodicity. The different realization of the RW periodicity plays a crucial role in the classification as a difference of the degeneracy of the free-energy. In our approach for the investigation of the confinement-deconfinement transition, we focus on the response of the system against $\theta$ as an indicator of the non-trivial free-energy degeneracy: The system does not show singularities along $\theta$ at $\left(T, \mu_{\mathrm{R}}\right)$ in the confined phase, but it shows the singularity in the deconfined phase. Details of singularities are explained in Sec. 2; for example, see Fig. 1. The confinement-deconfinement transition temperatures determined by the non-trivial free-energy degeneracy and the Polyakov-loop are matched with each other in the infinite quark mass limit. In the next subsection, we propose a new quantum order parameter of the confinement-deconfinement transition based on the RW periodicity. In the following discussions, we mainly concentrate on the case with $\mu_{\mathrm{R}}=0$.

\section{Definition of quark number holonomy}

The quark number density $\left(n_{q}\right)$ above $T_{\mathrm{RW}}$ should have the gap at $\theta=(2 k-1) \pi / N_{\mathrm{c}}$ which reflects the $\theta$-odd property. The schematic behavior of $n_{q}$ with $N_{\mathrm{c}}=3$ is shown in Fig. 1 . The periodic solid and dashed lines represents $n_{q}$ at sufficiently high and low $T$ comparing with $T_{\mathrm{RW}}$, respectively. By using $n_{q}$, we can construct the quantum order-parameter;

$$
\Psi(T)=\left[\oint_{0}^{2 \pi}\left\{\operatorname{Im}\left(\left.\frac{d \tilde{n}_{q}}{d \theta}\right|_{T}\right)\right\} d \theta\right]
$$

where $\tilde{n}_{q}$ is the normalized quark number density defined as $\tilde{n}_{q} \equiv C n_{q}$ here the coefficient $C$ $\left[\mathrm{MeV}^{-3}\right]$ is introduced to make $\tilde{n}_{q}$ dimensionless. It becomes non-zero at $T \gg T_{\mathrm{RW}}$ and zero at $T \ll T_{\mathrm{RW}}$ because the information of the gap at $\theta=(2 k-1) \pi / N_{\mathrm{c}}$ is missed through the differential calculus and the numerical integration. We call Eq. (2.1) the quark number holonomy.

The integrand of Eq. (2.1) is nothing but the quark number susceptibility $\left(\chi_{q}\right)$ at finite $\theta$. The expected behavior of the quark number holonomy as a function of $T$ is shown in Fig. 2. We assume that the RW endpoint is the second (first) order in the case A (B). The schematic phase diagram in the case $\mathrm{B}$ is shown in the inset figure of Fig. 2. When $T_{\mathrm{RW}}$ is the first-order, the RW endpoint 


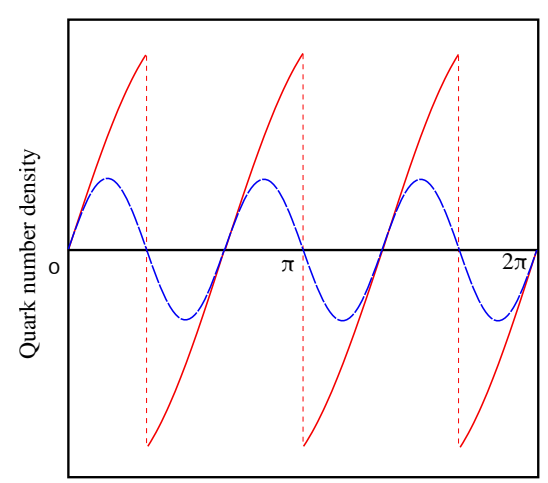

Dimensionless imaginary chemical potential

Figure 1: The schematic behavior of $n_{q}$ as a function of $\theta$ for $N_{\mathrm{c}}=3$. The periodic solid and dashed lines represent the quark number density at $T \gg T_{\mathrm{RW}}$ and $T \ll T_{\mathrm{RW}}$, respectively.

can have two more first-order lines starting from the RW endpoint. In this paper, we call it beard line and the endpoint temperature of the beard line is denoted by $T_{\text {Beard }}$. This triple point scenario has been predicted by the lattice QCD simulations $[8,9]$. This behavior may be induced by the correlation between the chiral and deconfinement dynamics, but details are still under debate.

In the case A where the RW endpoint is the second order, the quark number holonomy can be expressed as

$$
\Psi= \pm 2 N_{\mathrm{c}} \lim _{\varepsilon \rightarrow 0}\left[\operatorname{Im} \tilde{n}_{q}\left(\theta=\theta_{\mathrm{RW}}^{\mp}\right)\right]
$$

where $\theta_{\mathrm{RW}}^{\mp}=\theta_{\mathrm{RW}} \mp \varepsilon=\pi / N_{\mathrm{c}} \mp \varepsilon$ with the positive infinitesimal value $\varepsilon$. Below $T_{\mathrm{RW}}, n_{q}\left(\theta=\pi / N_{\mathrm{c}}\right)$ is exactly zero and thus $\Psi=0$, but $\Psi$ becomes non-zero above $T_{\mathrm{RW}}$. The coefficient $N_{\mathrm{c}}$ in Eq. (2.2) reflects the number of the gapped point in the $0 \leq \theta \leq 2 \pi$ region.

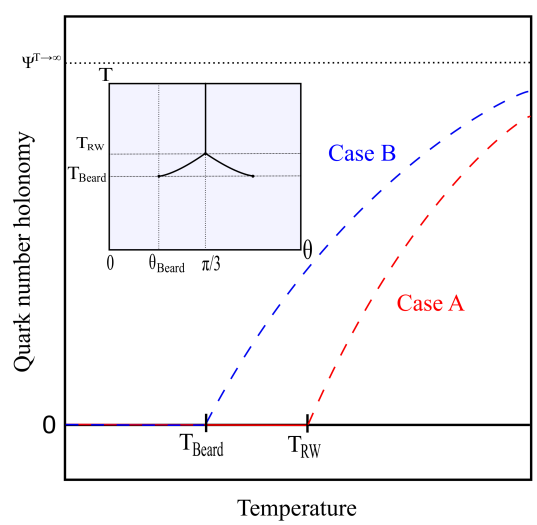

Figure 2: The expected behavior of $\Psi$ as a function of $T$. In the case A, the RW endpoint is the second order, while it is the triple point in the case B. The actual value of $\Psi^{T \rightarrow \infty}$ is explained in the text. The inset figure shows the schematic phase diagram in the case B with $N_{\mathrm{c}}=3$ as a function of $\theta$ and $T$. 
In the case B where the RW endpoint is the triple point, situations become complicated in the $T_{\mathrm{Beard}}<T<T_{\mathrm{RW}}$ region. In this region, $\tilde{n}_{q}$ jumps from high- $T$ curve to the low- $T$ curve at $\theta=\theta_{\mathrm{B}}$ where $\theta_{\mathrm{B}}$ is defined as the dimensionless imaginary chemical potential on the beard line. The quark number holonomy can be expressed as

$$
\Psi=2 N_{\mathrm{c}} \lim _{\varepsilon \rightarrow 0}\left[\operatorname{Im}\left\{\tilde{n}_{q}\left(\theta=\theta_{\mathrm{B}}^{-}\right)-\tilde{n}_{q}\left(\theta=\theta_{\mathrm{B}}^{+}\right)\right\}\right] \neq 0,
$$

where $\theta_{\mathrm{B}}^{\mp}$ mean $\theta_{\mathrm{B}} \mp \varepsilon$. The number of the gapped point is $2 N_{\mathrm{c}}$ in the $0 \leq \theta \leq 2 \pi$ region above $T_{\text {Beard }}$. Below $T_{\text {Beard }}, \Psi$ should be zero.

Some asymptotic behaviors such as the high $T$ and heavy $m$ behaviors can be obtained from the behavior of $n_{q}$. Details are shown in Ref. [7].

\section{Discussions}

Firstly, we discuss the current status of the present determination and the ordinary determination of the deconfinement temperature. Readers may doubt the validity of the present definition of the deconfinement transition temperature since the deconfinement temperature, $T_{\mathrm{D}} \equiv T_{\mathrm{RW}}$ or $T_{\text {Beard }}$, is substantially higher than the chiral pseudo-critical temperature. It was considered that the chiral and the deconfinement crossover take place at similar temperatures from the rapid change of the chiral condensate and the Polyakov-loop in the $2+1$ lattice QCD simulations; see for example Ref. $[10,11]$. With the highly improved quark action, it now seems that the Polyakov-loop grows very gradually $[12,13]$. An effective model analysis of recent lattice data implies that the deconfinement pseudo-critical temperature $(\sim 215 \mathrm{MeV})$ is substantially higher than the chiral pseudo-critical temperature [14]. By comparison, a recent lattice determination of the RW endpoint temperature with physical quark masses implies that the continuum extrapolated value of $T_{\mathrm{RW}}$ is 208(5) MeV [15]. Therefore, higher $T_{\mathrm{D}}$ does not invalidate the discussion, but may be supported by the recent lattice data via effective model analysis.

Secondly, we discuss the difference between the quark number holonomy and the dual quark condensate [16]. The dual quark condensate is defined as

$$
\Sigma^{(n)}=-\oint_{0}^{2 \pi} \frac{d \varphi}{2 \pi} e^{-i n \varphi} \sigma(\varphi)
$$

where $\varphi=\theta+\pi$ specifies the boundary condition for the temporal direction of quarks, $\sigma(\varphi)$ is the $\varphi$-dependent chiral condensate and $n$ represents the winding number along the temporal direction. Particularly, $\Sigma^{(1)}$ shares similar properties with $\Phi$ because $\Phi$ is also the winding number 1 quantity and thus it can be used as the indicator of the confinement-deconfinement transition. In the quenched approximation, the dual quark condensate is well defined, but there is the uncertainty in the dynamical quark case. Therefore, there is the uncertainty in the determination of the dual quark condensate. Also, it is well known that the dual quark condensate is strongly, for example, see Ref [17]. On the other hand, the quark number holonomy (2.1) can provide non-zero value above $T_{\mathrm{RW}}$ or $T_{\text {Beard }}$ without any uncertainties. It is the advantage of the quark number holonomy.

Finally, the sign problem is discussed when we calculate the quark number holonomy at finite $\mu_{\mathrm{R}}$. At finite $\mu_{\mathrm{R}}$, Eq.(2.1) should be replaced as $\Psi(T) \rightarrow \Psi\left(T, \mu_{\mathrm{R}}\right)$. This means that the $\theta$ integration should be evaluated with fixed $T$ and also $\mu_{\mathrm{R}}$. Therefore, we must consider the complex 
chemical potential where the sign problem arises. At finite imaginary chemical potential $\left(\mu_{\mathrm{R}}=0\right)$, we can use the $\gamma_{5}$ hermiticity and then the sign problem does not matter at finite imaginary chemical potential, $\mu^{*}=-\mu$ when we calculate $\Psi(T)$. On the other hand, at finite real chemical potential, the Lefschetz thimble path integral method [18] can help us. In Ref. [19], it is shown that this method leads the saddle-points which manifests the $\mathscr{C} \mathscr{K}$ symmetry where $\mathscr{C}$ and $\mathscr{K}$ express the charge and the complex conjugation operator, respectively. Unfortunately, the $\mathscr{C} \mathscr{K}$ symmetry is not preserved at finite complex chemical potential, $\mu_{\mathrm{R}} \neq 0$ and $\theta \neq 0$, when we calculate the quark number holonomy. Thus, the calculation becomes complicated even in the mean-field calculation of the QCD effective models. In this case, we should perform the matter-of-fact calculation based on the Lefschetz thimble path integral method. Actual challenge of the calculation will be shown elsewhere.

\section{Summary}

In this talk, we explained a new quantity to describe the confinement-deconfinement transition based on topological properties of QCD in the imaginary chemical potential region. We call it the quark number holonomy which is defined by the contour integral of the quark number susceptibility along the closed loop of $\theta$. The quark number holonomy seems to be similar to the Uhlmann phase which can be used to classify the topological order at finite $T$ in the condensed matter physics.

The quark number holonomy can have a non-zero value above $T_{\mathrm{RW}}$ or $T_{\mathrm{Beard}}$ and it becomes zero below these temperatures. This behavior is related with the different realizations of the freeenergy degeneracy above and below $T_{\mathrm{RW}}$. From the model independent analysis, we find that the quark number holonomy is proportional to $N_{\mathrm{c}}^{2}$ in the deconfined phase, while it does not in the confined phase if we determined the confinement-deconfinement temperature as the topological phase transition.

We have discussed the similarity between the quark number holonomy and the dual quark condensate which is sometimes used to investigate the confinement-deconfinement transition. Calculations of the dual quark condensate has the uncertainty when the dynamical quark is taken into account, but the quark number holonomy does not have such uncertainty. This is the strong advantage of the quark number holonomy. Finally, we have discussed the sign problem when we calculate the quark number holonomy at finite real chemical potential. In this case, we should consider the complex chemical potential and thus we need extremely care of the sign problem.

\section{References}

[1] X.G. Wen. Topological Order in Rigid States. Int.J.Mod.Phys., B4:239, 1990.

[2] Masatoshi Sato. Topological discrete algebra, ground state degeneracy, and quark confinement in QCD. Phys.Rev., D77:045013, 2008.

[3] Kouji Kashiwa and Akira Ohnishi. Topological feature and phase structure of QCD at complex chemical potential. Phys. Lett., B750:282-286, 2015.

[4] Andre Roberge and Nathan Weiss. Gauge Theories With Imaginary Chemical Potential and the Phases of QCD. Nucl.Phys., B275:734, 1986. 
[5] A. Uhlmann. Parallel Transport and Quantum Holonomy along Density Operators. Rep. Math. Phys., $24: 229,1986$.

[6] O Viyuela, A Rivas, and MA Martin-Delgado. Uhlmann Phase as a Topological Measure for One-Dimensional Fermion Systems. Phys.Rev.Lett., 112:130401, 2014.

[7] Kouji Kashiwa and Akira Ohnishi. Quark number holonomy and confinement-deconfinement transition. Phys. Rev., D93(11):116002, 2016.

[8] Massimo D'Elia and Francesco Sanfilippo. The Order of the Roberge-Weiss endpoint (finite size transition) in QCD. Phys. Rev., D80:111501, 2009.

[9] Claudio Bonati, Guido Cossu, Massimo D’Elia, and Francesco Sanfilippo. The Roberge-Weiss endpoint in $N_{f}=2$ QCD. Phys.Rev., D83:054505, 2011.

[10] Y. Aoki, Z. Fodor, S. D. Katz, and K. K. Szabo. The QCD transition temperature: Results with physical masses in the continuum limit. Phys. Lett., B643:46-54, 2006.

[11] M. Cheng et al. The QCD equation of state with almost physical quark masses. Phys. Rev., D77:014511, 2008.

[12] Szabolcs Borsanyi, Zoltan Fodor, Christian Hoelbling, Sandor D Katz, Stefan Krieg, Claudia Ratti, and Kalman K. Szabo. Is there still any $T_{c}$ mystery in lattice QCD? Results with physical masses in the continuum limit III. JHEP, 09:073, 2010.

[13] A. Bazavov et al. The chiral and deconfinement aspects of the QCD transition. Phys. Rev., D85:054503, 2012.

[14] Akihisa Miyahara, Yuhei Torigoe, Hiroaki Kouno, and Masanobu Yahiro. Equation of state and transition temperatures in the quark-hadron hybrid model. 2016.

[15] Claudio Bonati, Massimo DElia, Marco Mariti, Michele Mesiti, Francesco Negro, and Francesco Sanfilippo. Roberge-Weiss endpoint at the physical point of $N_{f}=2+1$ QCD. Phys. Rev., D93(7):074504, 2016.

[16] Erek Bilgici, Falk Bruckmann, Christof Gattringer, and Christian Hagen. Dual quark condensate and dressed Polyakov loops. Phys.Rev., D77:094007, 2008.

[17] Sanjin Benič. Physical interpretation of the dressed Polyakov loop in the NambuâĂŞJona-Lasinio model. Phys.Rev., D88(7):077501, 2013.

[18] Edward Witten. Analytic Continuation Of Chern-Simons Theory. AMS/IP Stud. Adv. Math., 50:347-446, 2011

[19] Yuya Tanizaki, Hiromichi Nishimura, and Kouji Kashiwa. Evading the sign problem in the mean-field approximation through Lefschetz-thimble path integral. Phys. Rev., D91(10):101701, 2015. 